\title{
Nuevos desafíos, renovadas miradas: algunas propuestas al debate sobre género, desarrollo y turismo en el Pacífico mexicano
}

DOI: $10.32870 /$ mycp.v13i37.338

Ana Bergareche ${ }^{1}$

Esperanza Vargas ${ }^{2}$

\section{Resumen}

1 presente trabajo es parte de una investigación más amplia, actual-
mente en proceso, sobre género, trabajo y empoderamiento en la
localidad turística de Puerto Vallarta, Jalisco, México. Los objetivos del ensayo son: 1. Ofrecer una panorámica teórica del debate sobre género, trabajo y desarrollo y sus implicaciones en el contexto del turismo; 2. Como parte de dicha panorámica, establecer una relación entre género, trabajo y empoderamiento en este contexto, definiendo el empoderamiento como la capacidad de negociación hacia el bienestar individual, tomando en cuenta tanto los aspectos objetivos como los subjetivos; 3. Plantear ciertos cuestionamientos a la problemática en cuestión, con el fin de poder enriquecer y reorientar dicho debate hacia diferentes vertientes que ofrezcan alternativas de cambio social y empoderamiento a las mujeres trabajadoras del turismo en la Cuenca del Pacífico.

Con este propósito, en primer lugar, se señalará cómo la ausencia de un enfoque de género en la aplicación de programas y proyectos en el contexto

1. Profesora investigadora en el Centro Universitario de la Costa (cUc), Puerto Vallarta, Universidad de Guadalajara. Doctora en género y desarrollo (2001), Universidad de Londres, Londres. Correo electrónico: ane4@yahoo.com.

2. Profesora docente en el Centro Universitario de la Costa (CUC), Puerto Vallarta, Universidad de Guadalajara. Candidata al Doctorado en Desarrollo Sustentable de Cedestur (CUC). Correo electrónico: esperanzavgas@hotmail.com. 
del turismo, así como en la definición del mismo, ha parcializado las visiones el desarrollo en este sector. En segundo lugar, se revisarán las diversas perspectivas sobre la incorporación de la mujer al trabajo en el contexto del desarrollo con algunas referencias al turismo, haciendo hincapié en la necesidad de ir más allá de los aspectos estructurales de la desigualdad de género para poder identificar avenidas de poder tangibles para las mujeres trabajadoras en este contexto. Finalmente, se propondrá un enfoque dentro del mismo debate que explore el rol laboral de las mujeres en relación a la cultura local, las emociones y el aspecto simbólico del trabajo, elementos en sí conectados con las relaciones de género en el hogar. De esta manera, se podrán identificar avenidas de empoderamiento desde el ámbito individual y cotidiano que ofrezcan alternativas reales hacia una mejor calidad de vida para las mujeres trabajadoras de nuestro entorno local.

\section{Abstract}

The present work is part of a broader research project, presently in process, about gender, work and empowerment in the touristic town of Puerto Vallarta, Jalisco, Mexico. The essay's objectives are: 1) To offer a theoretical view of the origins and contributions of the debate on gender, work and empowerment and its relation to tourism; 2) As part of this view, to establish a relationship between gender, work and empowerment in this context, defining empowerment as the ability to negotiate towards individual well being, considering objective and subjective elements of negotiation; 3) To question some of the premises within the debate in order to enrich and reorient it towards different alternatives of social change and empowerment for women workers in the Pacific area.

For this purpose, it first focuses on how the absence of gender in the application of programs and projects in the context of tourism, as well as in its definition, has generated a partialized view and limited development trends in this sector. Secondly, it revises the various perspectives on women's work in this context, focusing on the need to move beyond structural aspects of gender inequality in order to identify avenues of power that are tangible for women workers. Finally, it proposes a focus that explores the aspects of work that relate to local culture, emotions and the symbolic aspects of labor, all of them in connection to gender power relations within the household. This perspective could unfold elements of empowerment from an individual 
and day-to-day standpoint that offer plausible alternatives to women workers and a better quality of life for them at the local level.

\section{El eslabón perdido: género y turismo en el contexto de la globalización}

La entrada de las mujeres al espacio público en el contexto del turismo no es reciente; sin embargo, es notoria la ausencia de aportaciones académicas a este fenómeno en el contexto del Pacífico mexicano, así como de sus efectos sociales, económicos y culturales. No cabe duda de que éstos han sido significativos, impactando las biografías de mujeres, hombres y familias y modificando sus identidades tanto en los espacios privados como públicos. Sin embargo, la escasez de literatura sobre el tema hace que las preguntas sigan siendo más numerosas que las respuestas, cuestionando las premisas, las políticas, y la ideología que han ido marcando las relaciones entre los géneros en diferentes etapas del turismo.

La atención dirigida al turismo en el contexto global es sobresaliente en relación a otros sectores económicos, convirtiéndola en un medio idóneo para lograr la sustentabilidad; su desarrollo económico ha sido más documentado y atendido que los procesos sociales y ambientales. Las cifras acerca del crecimiento de esta industria y de sus posibilidades económicas apuestan hacia el desarrollo local, nacional e internacional. Es este último el que se lleva la mayor tajada, disfrutando de más de dos tercios de los ingresos del turismo que nunca llegaron a la economía local debido a la gran fuga de divisas (Organización Internacional del Trabajo, 2001).

La centralización y competencia de esta industria la hace tierra exquisita para la inversión extranjera, iluminando el camino para evaluar los impactos del proceso globalizador que la enmarcan. El proyecto liberal de desarrollo, donde la fuerza y poder del Estado pierden protagonismo, está sembrado y creciendo en los destinos turísticos. La eliminación de barreras que limiten su crecimiento está siendo una prioridad para las agencias internacionales, promoviendo la expansión y mejora de las infraestructuras locales.

A pesar del tono optimista de estas iniciativas, el sueño del desarrollo empezó a esfumarse cuando se evidenciaron las consecuencias sociales y ambientales de las mismas. Entre ellas figura una dolarización de los destinos (una forma encubierta de devaluación local), que empobrece a los habitantes que utilizan la moneda nacional y abarata artificialmente los costos para los 
extranjeros. Así mismo, la transnacionalización del mercado interno, con el asentamiento de establecimientos comerciales, devasta la estructura del pequeño comercio local y con ello el campo laboral, ya que contribuye al abandono de las pautas de producción tradicionales. Finalmente, el ambiente relajado que suele asociarse con los destinos exóticos fomenta el desarrollo del comercio sexual, cuyas principales víctimas son mujeres y niños (OIT, 2001).

La complejidad que acompaña los procesos del turismo y la unidireccionalidad con que ha sido atendido e interpretado, apostando sobremanera al desarrollo económico, ha propiciado un debate público que intenta corregir, aminorar y redefinir el rumbo que ha seguido esta industria (Salazar, 2006; Byrne, 2005). Una de las limitaciones en la investigación sobre turismo y su vinculación con el desarrollo se deriva del escaso valor que se les ha otorgado a los actores sociales inmersos en los procesos productivos. Los estudios en este sector han marcado una excesiva centralidad en el análisis de la producción, de tal forma que los actores se tornan invisibles y sólo aparecen cuando se les involucra en aspectos organizacionales o como fuerza de trabajo funcional (multicalificación, rotación de trabajo, versatilidad, etc.), o en su aspecto numérico y en relación con las políticas laborales (nuevos esquemas salariales, de horario y contratación), además de algunas formas atípicas de empleo (De la O. Martínez, 2000; Pérez, 1996; Villaseñor, 1993).

Una propuesta para solventar dichas ausencias consiste en integrar la perspectiva de género a la investigación sobre el turismo, ya que el área social del turismo no está ajena a las relaciones de poder que involucra. Tal como lo puntualiza Moreno y Lunar (2006: 378), este enfoque permite: “...evidenciar como los grupos humanos a partir de las diferencias biológicas, construyen los conceptos de feminidad y masculinidad y atribuyen simbólicamente características, posibilidades de actuación y valoración diferentes a las mujeres y a los hombres, produciendo en la mayoría de las sociedades sistemas sociales no equitativos...". Un ejemplo de esta perspectiva se encuentra en el análisis de las prácticas y mitos turísticos.

Los turistas no sólo ejercen una contemplación voyeurista del mundo, sino que ayudan a re-imaginarlo y refabricarlo, en consonancia con sus diversos prejuicios de género (Cheong y Miller, 2000, citados en Salazar, 2006; Bishop y Robinson, 1998). Estudiar la interdependencia entre el poder y la resistencia en este contexto puede ayudarnos a la comprensión de los procesos de cambio que se gestan en las comunidades destino, iluminando vetas que deben ser atendidas para lograr un desarrollo social sostenible. 
Kinnaird y Hall (1996) integran herramientas conceptuales y metodológicas para la comprensión de las relaciones sociales y el comportamiento de grupos e individuos en este contexto. Su base conceptual para la relación entre género, turismo y desarrollo se resume en los siguientes puntos: 1. Los procesos de desarrollo turístico y las actividades relacionadas con el turismo surgen de sociedades determinadas por el género; 2. Las relaciones de género configuran y a la vez son configuradas por las prácticas de todas las sociedades; 3. Las relaciones de poder que rodean los procesos de desarrollo turístico representan una prolongación de las políticas de las relaciones de género. Este modelo nos da una herramienta para poder integrar la perspectiva de género en los procesos de turismo y desarrollo.

\section{Caminos recorridos y otros por recorrer: evolución y propuestas al debate sobre género y desarrollo en su relación con el turismo}

Dentro del debate sobre género y desarrollo se encuentra el cuestionamiento sobre los efectos que el trabajo remunerado de las mujeres ha tenido en sus vidas. Esta vertiente analítica tiene distintas bases teóricas, ligadas a la interpretación de los distintos paradigmas del desarrollo. Una de ellas es la teoría de la modernización (modelo dominante a partir de la II Guerra Mundial), postulando que las regiones en desarrollo deben seguir los pasos a los países desarrollados.

En función de esta premisa se prioriza la transferencia de tecnología moderna hacia los países en desarrollo. Se concede también gran importancia a la industrialización,

Los turistas no sólo ejercen una contemplación voyeurista del mundo, sino que ayudan a re-imaginarlo y refabricarlo, en consonancia con sus diversos prejuicios de género considerándola como elemento fundamental de la modernización y aspecto imprescindible del desarrollo (Hidalgo, 1998; Kabeer, 2004; López y Alcalde, 1999). Finalmente, los asuntos de las mujeres y de otros grupos vulnerables fueron considerados como un problema de "integración" en el proceso de desarrollo que encontraría su solución conforme éste fuera avanzando.

Sin embargo, a pesar de reconocer a las mujeres como protagonistas fundamentales del desarrollo, se les siguió considerando como vulnerables y 
beneficiarias. De ahí que las políticas se enfocaran en dar respuesta a las necesidades prácticas de las mujeres, como la ayuda alimentaria, la nutrición y la planificación familiar y no en aspectos de prevención como los programas de educación comunitarios (De Villota, 1999; Moser, 1995). Como consecuencia, las mujeres sólo podían acceder a apoyos bajo un paradigma que reforzaba el modelo del ama de casa (Moira, 2004; López y Alcalde, 1999).

Otras reflexiones similares llevan al surgimiento de la perspectiva MED (mujeres en el desarrollo) cuyo enfoque es la integración de la mujer al proceso de desarrollo, y que fue adoptada por las Agencias Especializadas de Naciones Unidas y por la Década de las Mujeres (1975-1985), teniendo su influencia en la cooperación internacional de países donantes, en particular la USAID (United States Agency for International Development). Dentro de esta corriente de pensamiento, la eliminación de las desigualdades entre hombres y mujeres fue aceptada como un objetivo evidente, que tenía sentido tanto en la promoción de un desarrollo más eficaz como en la defensa de los derechos de las mujeres. Estas medidas cambiaron los patrones de participación de la mujer en la fuerza de trabajo, haciendo hincapié en la visibilidad e integración, para solventar la marginalidad de las mujeres en el desarrollo y los estereotipos y prejuicios de los planificadores (Monreal, 1999; Kabeer; 2004).

Respecto a las implicaciones para el trabajo de la mujer en el contexto del turismo, esta perspectiva alude a una ampliación de oportunidades laborales con una mejoría en las condiciones de vida de las mujeres. Así mismo, la combinación de la educación formal con el salario, así como el compromiso que las mujeres asumirían con el trabajo, pronosticaba relaciones de género igualitario. De esta manera, se consideró el control de los recursos económicos como un elemento que podía traer una mayor participación de las mujeres en la toma de decisiones familiares y una distribución más igualitaria de las labores domésticas, sobre todo en las clases medias y altas (Casique, 2007; Beneria, 2005).

Asimismo, esta teoría argumenta que la migración ofrece enormes posibilidades para las mujeres, apostando a una posición económica y un interés político comunes pese a las fronteras nacionales, realzando el mito del capitalismo como portador de interdependencia y equidad a nivel de la escala planetaria (Pérez, 1996; Falquet, 2003; Bauman, 1999; Velázquez, 1993). De acuerdo a dicha perspectiva, esto implicaría la redefinición de roles y funciones al interior de las familias, así como la aparición de otras pautas de género. 
El movimiento MED intenta conciliar con el discurso oficial sobre el desarrollo sin cambiar la construcción social de las relaciones entre hombres y mujeres. Entre sus logros podemos destacar que hizo visible la aportación económica de los países de la periferia, se cuestionó la neutralidad del desarrollo y buscó la integración entre los sexos mediante una mayor y mejor participación de las mujeres en la actividad económica del mercado (Boserup, 1970; Rendón, 2003; Pérez, 1996). Sin embargo, al señalar la integración como requisito del desarrollo, se dio por sentado que las mujeres no estaban integradas. Por tanto, se puede interpretar que los espacios privados, históricamente asignados a las mujeres, son minimizados, vistos como irrelevantes y sin poder, descalificando las labores domésticas y emocionales — cuidado, valoración, amor - que se producen y reproducen en esos espacios.

Cuatro décadas más tarde se descarta la hipótesis de que el confinamiento de las mujeres en la esfera privada era la causa de su baja jerarquía sociopolítica, dado que éstas ya se establecieron en la esfera pública y la igualdad prometida por la modernidad no se ha hecho notar. Según el último informe presentado por The Global Gender Gap Report (2007) México ocupa el lugar 75 de 115 en la lista de países evaluados en materia de equidad de género. En el Índice de Equidad de Género, Avances y Retrocesos (2008), donde se evalúa la brecha de empoderamiento, educación y economía, se observa que: 1. La brecha de empoderamiento es de $38.2 \%$, este dato contempla el porcentaje de mujeres que ocupan cargos técnicos, de dirección, gobierno, mujeres parlamentarias y cargos ministeriales; 2 . La brecha económica es de 44.3\%; y 3. La brecha educativa es de $97.3 \%$.

Las críticas feministas al enfoque MED, desde la perspectiva del Género en el Desarrollo (GED), son que el modelo mismo de desarrollo no es cuestionado sino el hecho de que las mujeres no se beneficien de él. No se cuestiona el mercado como medio y fin, ni se evidencian los intereses económicos e ideológicos de sus argumentos (Bergareche, 1997, 2001). La puntualización que hace el feminismo liberal en promover la integración de las mujeres como antesala de su liberación ha tenido a menudo efectos negativos no tanto por sus intenciones, sino por marginar las relaciones socialmente definidas entre los hombres y las mujeres en cada contexto socioeconómico.

Estas reflexiones se dirigen a las maneras en que mujeres y hombres están integrados de manera desigual al desarrollo, debido al entretejido de relaciones de clase, género y etnia que lo conforman en los niveles local y global (Kabeer, 2004; López y Alcalde, 1999; Rendón, 2003; Marrero, 2005). La adopción de 
un enfoque de género como lente alternativo contribuiría a analizar, evidenciar y potenciar los recursos que surgen en las mujeres teniendo como antesala su participación laboral.

El enfoque GED en el turismo priorizó las desigualdades de género en relación a individuos, grupos, discursos sociales, tipos de turismo y naciones (Byrne, 2005; Bishop y Robinson, 1998). Desde esta perspectiva, pretende integrar los procesos de configuración y las múltiples dimensiones del trabajo donde la mujer participa. Su base teórica se encuentra en el feminismo marxista, el cual se refiere a las estructuras de poder capitalistas como fundamento de la desigualdad de género en el trabajo.

Esta perspectiva está abanderada por las perspectivas de la explotación y la marginación, que interpretan el papel femenino en el desarrollo como explotación y exclusión. La perspectiva de la explotación sostiene que la causa de la desigualdad estriba en un sistema capitalista que se beneficia de la opresión de la mujer. Su análisis acentúa la explotación más que el prejuicio sexista, la estructura más que los individuos que actúan en su seno, y más específicamente los beneficios materiales que el capitalismo obtiene de la posición y la función de la mujer (Arriagada, 1994; Romero, 2000; Gatens, 2004).

En definitiva, se destaca la funcionalidad del trabajo femenino (en especial el familiar no remunerado) y del modelo tradicional de familia nuclear para la acumulación capitalista. En la medida en que el trabajo de la mujer en el ámbito familiar permite absorber parte de los costos de producción de la fuerza de trabajo, deprime los salarios y asegura a los empresarios márgenes más elevados de ganancia. La funcionalidad del trabajo de la mujer sirve a la clase dominante, ya que por su baja calificación y el acondicionamiento de la esfera doméstica, constituye una mano de obra barata a la que se puede explotar y ubicar en espacios ocupacionales segregados y peor pagados.

Al mismo tiempo, el mantenimiento de las necesidades domésticas del proveedor es absorbido por el "ama de casa", y su valor productivo oscurecido por justificaciones morales y biológicas. De esta manera, la mujer se asume como una cuidadora innata de la familia, tanto por la sociedad como por ella misma. Sin embargo, la historia nos indica que las mujeres siempre han trabajado, y que ése ha sido su estado natural, más que la imagen de ama de casa (García, 2004). Desde esta óptica, capitalismo y patriarcado se refuerzan necesariamente (Tiano, 1994, citado en Bergareche, 2004; Romero, 2006).

Una implicación significativa para la identidad laboral de la mujer es que el peso social del rol de ama de casa hace que no se asuma como traba- 
jadora asalariada una vez que ejerce un empleo fuera del hogar (Bergareche, 2001, 2006). Al mismo tiempo, considera la participación del hombre en las funciones domésticas como una ayuda que nace de su buena voluntad, ratificando así su posición. En la misma línea se argumenta que su integración al desarrollo le ocasiona una pérdida relativa de estatus social, abriendo las diferencias de género al devaluar las actividades que realizan y restringiendo las opciones económicas disponibles (Boserup, 1990 citado en De Oliveira y Ariza, 1999).

Investigaciones realizadas a partir de los años noventa sobre las prácticas del turismo han evidenciado que la construcción social de la sexualidad es otro elemento utilizado por el capitalismo para obtener beneficios. El argumento se centra en que el lenguaje de la promoción suele privilegiar la mirada masculina y heterosexual. De este modo, el mercado exaltado por el género recrea mitos y fantasías masculinas particulares de una experiencia seductora y sexualizada (Bishop y Robinson, 1998). Aitchison (2001) y Enloe (1999) argumentan que el turismo está asociado con lo exótico y lo erótico como parte del móvil del turismo y del consumo global.

Se observa cómo el material publicitario destinado a promover el turismo de playa usa la imagen de la mujer joven, esbelta y con escasa ropa, aventurera y atrevida. La ausencia de la variable género en esta perspectiva colabora con estas prácticas, evadiendo o descalificando el componente social simbólico e ideológico donde se construyen, así como el poder que las permea (Byrne, 2005; Kinnaird y Hall, 1994). Así, el escenario laboral del turismo aprovecha la construcción de las identidades de género que el patriarcado perpetúa y reproduce los modelos de relación genérica.

Esta dinámica impide que los individuos tengan las mismas posibilidades de acceso a oportunidades personales y colectivas, observándose este aspecto en la estacionalidad de los trabajos desarrollados por hombres y mujeres, pero afectando particularmente a éstas últimas (Salazar, 2006; Falquet, 2003). Tal y como se señaló más arriba y como parte de la perspectiva de la explotación, los supuestos genéricos del capitalismo y el patriarcado se establecen en la identidad de hombres y mujeres y funcionan como argumentos de la polaridad genérica: a la mujer se le han adjudicado los elementos específicos al rol materno (proveedora emocional) y al hombre se le ha definido como el proveedor instrumental, responsable de obtener los recursos materiales necesarios para la manutención familiar y el éxito profesional (Walters et al., 1996; Medina, 2000; Corsi, 2003). 
Como consecuencia, se asume que el turismo trae escasos beneficios para las mujeres, ya que generalmente no son inversionistas ni tienen acceso a los mejores empleos (Pérez, 1996; Falquet, 2003). Otros estudios analizan la violencia sexual que las trabajadoras de este sector tienen que enfrentar de los turistas del sexo masculino (Falquet, 2003). Además, la subida de precios provocada por el turismo, el empobrecimiento que supone el despojo de sus recursos tradicionales (pesca, agricultura) y la incitación activa por parte de los turistas provoca casi inevitablemente el desarrollo de la prostitución.

Las exigencias de flexibilizar el trabajo por parte de las empresas y el Estado han legitimado la discriminación sexual del trabajo, presentándolas como necesarias para la eficiencia social, siendo que los empleos "flexibles" a menudo generan situaciones de discriminación laboral (De la O Martínez, 2001). Los grupos más afectados por estas políticas son las mujeres, quienes aparecen como sujetos deseables debido al tipo de relación que mantienen con el mercado de trabajo. Debido a su construcción social como amas de casa constituyen una mano de obra barata a la que se puede explotar, ubicándola en espacios ocupacionales segregados con salarios inferiores a la media (Tiano, 1994; citado en Bergareche 2001).

En el contexto global, Enloe (2000) señala que $80 \%$ de los empleos en el turismo estarían ocupados por mujeres. Datos más recientes comparativos por sexo muestran el número de mujeres empleadas a nivel global por salario semanal y horas de trabajo (Oficina Internacional del trabajo, 2008). Aunque no se dispuso de datos segregados por género para todos los países y en el sector turístico, los resultados muestran un aumento en la participación de las mujeres en la industria, estimado por el crecimiento de la actividad en países específicos. También reflejan que las mujeres son significativas para la industria, siendo $46 \%$ de la fuerza laboral (porcentaje más alto que el de la fuerza laboral en general, de $34 \%$ a $40 \%$ ).

Hay que tener en cuenta en estas proporciones que el aumento de las mujeres en el campo laboral no ha generado una reducción de horas dedicadas a las tareas domésticos y esto implica jornadas semanales de hasta de 70 a 90 horas. En cuanto a la relación entre las horas laborables de mujeres y hombres y sus respectivos salarios en los países en desarrollo, los salarios promedio de las mujeres disminuyen hasta $70 \%$ de los salarios de los hombres y es más probable que las mujeres sean empleadas con base en tiempo parcial que éstos. 
Lo anterior muestra que en el turismo se detectan significativas segregaciones por género, impactando de forma negativa la calidad de vida de las mujeres al tener que desempeñar varios roles y funciones que hacen más compleja su vida cotidiana y limitan su desarrollo personal. Estas limitaciones a menudo conducen a problemas de salud, falta de cuidado personal, obstáculos en el desarrollo profesional y en la calidad de su tiempo de ocio o ausencia del mismo, con efectos negativos en su capacidad de autonomía (Bergareche, 2003). Esto dista mucho de la meta propuesta por la Organización Mundial del Trabajo, que tiene como objetivo del desarrollo del milenio (ODM) promover la igualdad entre los géneros y la autonomía de la mujer (Boletín Hechos y Tendencias del Turismo, 2007).

El mercado laboral no ha roto los patrones de jerarquía sexual que según Beck (1988: 38) funcionan de la siguiente manera: “... cuanto más marginal se considera a un ámbito de tareas, cuanto menos influyente es un grupo, tanto mayor es la probabilidad de que las mujeres hayan conquistado en estos campos posibilidades de ocupación...” y en su versión inversa, “... cuando más central es un ámbito para la sociedad, cuanto más poderoso es un grupo, tanto menos representadas están las mujeres...".

Sin embargo, aun considerando la innegable discriminación de la mujer en el capitalismo global, la perspectiva de la explotación ha sido criticada con base en que la subordinación aparece como estática y sistemática. También se señala que esta premisa descuida la opresión de las mujeres en sociedades socialistas, a la vez que define a la mujer como víctima pasiva del sistema capitalista, siendo imposible su liberación (Romero, 2006).

Asimismo, al situar el análisis de la subordinación femenina en la esfera económica, desconoce otros ámbitos de interacción en que participan las mujeres, desde la arena política y la cultural, hasta los procesos de construcción de la subjetividad y la identidad (Bergareche, 2006). Una perspectiva menos extrema dentro del enfoque GED es la perspectiva de la marginalidad, la cual considera que la igualdad de género es factible a través de la acción política y de la transformación de las instituciones en el capitalismo. Este cambio de enfoque tiene implicaciones en la planificación y la implementación de políticas públicas.

La visión que ofrece el Banco Mundial en la última década (2006: 33) se puede entender como un esfuerzo para mejorar la posición de las mujeres, esperando un beneficio y una transformación paulatina de la sociedad: 
[...] las políticas de desarrollo que no toman en cuenta las cuestiones relativas al género y no consideran esas desigualdades, tendrán limitada su efectividad. Para promover la igualdad del género y la efectividad del desarrollo, se sugiere combinar una estrategia a largo plazo que se base en una reforma de las instituciones y establezca derechos y oportunidades iguales para mujeres y hombres, fomentando un desarrollo económico que ofrezca incentivos para más igualdad de recursos y participación, con medidas más eficientes que tengan en cuenta las desigualdades persistentes en esos aspectos. El Estado, los grupos de la sociedad civil y la comunidad internacional, todos deben jugar un papel decisivo en la lucha contra la discriminación de la mujer, permitiendo que las sociedades cosechen enormes beneficios.

Incorporar la dimensión de género a los planes y políticas de desarrollo implica la reconstrucción de su propia historia y el desarrollo de nuevas relaciones entre los sexos en el contexto del desarrollo. Resaltamos el punto de vista que Amartya Sen (2000: 16), tiene al respecto:

[...] el poder de las mujeres tiende a aliviar el descuido de los niños y la mortalidad, lo mismo que reducir la fertilidad y la sobrepoblación y, en términos más generales, tiende también a ampliar el alcance del interés y preocupación por los problemas sociales.

El desarrollo de las capacidades de las mujeres no sólo aumenta la libertad y el bienestar de éstas, tiene también efectos en la vida de todos.

Bajo un rubro más complejo dentro de la perspectiva de la marginación, Deere (1977, citado en De Oliveira y García, 1999) afirma que el desarrollo ha ocasionado tanto pérdidas como ganancias en la condición socioeconómica femenina. De acuerdo con esta autora, la mejoría económica no ha conducido a una elevación del estatus femenino, aunque sí otorga un mayor espacio de autonomía.

En términos generales, la transición hacia los enfoques GED ha cuestionado que el acceso de las mujeres al trabajo remunerado sea una condición suficiente para romper las barreras de género (De Villota, 1999). Se ha hecho visible el papel que juega la ideología de género como un filtro que orienta, estructura y dosifica el impacto del trabajo de las mujeres en sus vidas. La problemática central no es ya la integración de la mujer en el desarrollo, sino el de las estructuras, procesos y relaciones de poder que determinan la posición desventajosa de la mujer en diferentes sociedades. Se han extendido también las alternativas con que se argumentan los problemas y las solucio- 
nes, articulando dimensiones socioeconómicas, culturales y particularmente ideológicas.

Pero, más allá de defender una posición respecto a las perspectivas mencionadas o de ofrecer un mapa de la realidad de la mujer trabajadora, se pretende clarificar y puntualizar los supuestos o creencias bajo las que se rigen. La ausencia teórica que predomina en los paradigmas dominantes que abordan el desarrollo con enfoque de género es la escasa atención al conocimiento local y las dimensiones culturales (Bergareche, 2006), a la dimensión emocional y psico-afectiva (Vargas, 2009) y la ausencia de participación de los expertos en delinear políticas que coincidan y estén contextualizadas con la realidad cotidiana de mujeres, hombres y familias. Sin embargo, quedar atrapados en cualquiera de los argumentos anteriores obstaculiza la búsqueda de nuevas formas de posicionamiento de las mujeres frente a hechos que acompañan la globalización que parecen de imposible o difícil reversión (Melillo et al., 2004).

Como se puede advertir tras la breve revisión de las perspectivas teóricas, lo que está implícito en cada una de ellas son los profundos sesgos de las teorías sociopolíticas dominantes, los cuales pueden impedir que se adviertan rasgos de cotidianeidad en las relaciones de género en el contexto del turismo. Este aspecto es fundamental en su vertiente de clase, cultura, etnia y en relación a los elementos psicológicos de la experiencia de las mujeres, para adquirir una comprensión integral de su día a día y desarrollar las medidas adecuadas hacia el bienestar y la igualdad. Una actitud crítica y reflexiva que analice los compromisos discursivos latentes en los relatos de las mujeres impedirá que nos sometamos a los mandatos de un discurso abstracto y alejado de las voces cotidianas. Desde la expansión del saber es posible cambiar la posición del conocimiento y convertirnos en colaboradores y mediadores para después poder desarrollar una visión más anclada en la realidad y más efectiva para el cambio social.

Esta perspectiva nos alienta a investigar y a tomar en cuenta los procesos de empoderamiento y acción de las mujeres, distinguiendo cuáles son las estrategias de resistencia preexistentes y las emergentes en relación con el trabajo en el contexto del turismo. La tendencia desproporcionada que se le ha otorgado a los impactos negativos experimentados por parte de los pobladores locales en relación con el turismo puede cambiar, en la medida en que dejemos de asumir que el turismo se impone indefectiblemente a personas pasivas e impotentes. 
Algunos autores ya han descrito la manera en cómo el turismo internacional puede llegar a ser un vehículo empoderador de auto-representación. Los pobladores locales pueden sentirse empoderados por las interacciones con los turistas en sus procesos de auto-definición y en los aspectos de su identidad que desean poner en realce o minimizar (Cohen, 2002; Aitchison, 2001). En el actual clima de intercambio global económico y cultural ha cobrado importancia fundamental la búsqueda de la identidad y su reconstrucción. El turismo, tal como lo expresa Medina (2003), puede ofrecer los medios para reevaluar estos compromisos identitarios, facilitando la posibilidad de apropiarse del turismo y usarlo de manera simbólica para construir cultura, tradición e identidad.

\section{Poder, emociones e identidad: revistiendo el empoderamiento en el contexto del desarrollo y el turismo}

Las reflexiones anteriores nos llevan a puntualizar los procesos de empoderamiento como parte de los ejes centrales de GED. Las estrategias dirigidas hacia el empoderamiento cada día adquieren más importancia en los proyectos de desarrollo. La ampliación del entendimiento del poder ha permitido su reconocimiento más allá del control de los recursos físicos y financieros, abordando la manera en cómo la ideología determina reglas e ideales. Desde su aspecto social, el enfoque GED destaca que toda planeación debe partir de un análisis de las relaciones sociales enraizadas en las principales instituciones, llámese el Estado, el mercado, la comunidad o el hogar (Kabeer, 2004). De ahí la importancia de empoderar a las mujeres, dotándolas de herramientas para aumentar su poder de negociación y posibilitar la redistribución del poder en las relaciones de género y en la toma de decisiones.

Trasladado al área individual y psicológica, el ejercicio de poder no sólo les impide a las personas hacer las cosas sino también pensarlas. Este ámbito del poder encuentra una expresión variable e inestable en las redes y alianzas que permean los espacios de la vida (Payne, 2002; Droeven et al., 1997). En cuanto a las relaciones de género dentro de este contexto, reiterativamente se escucha en las conversaciones cotidianas cómo la sumisión, el sacrificio y el sufrimiento silencioso son virtudes, convirtiéndose en un ideal colectivo de la feminidad. Esta forma de "poder", desde la perspectiva de las nociones tradicionales sobre el género, se convierte en una manera eficiente de opresión que generalmente permanece oculta y escasamente se analiza (White, 
1994; White y Epston, 1993). Sus efectos se dejan ver en el mantenimiento de relaciones asimétricas, condicionando el sentido de libertad y la elección de las mujeres.

Estudiar el empoderamiento como un elemento significativo del proceso de cambio individual en el contexto del desarrollo y el turismo, nos invita a tocar avenidas del poder a través del trabajo poco estudiadas y no tangibles: la autoestima, la conciencia, el control percibido y las áreas subjetivas de la competencia y la eficacia. Si estos procesos llegan a formar parte del repertorio psicológico de las mujeres trabajadoras, es muy probable que el peso de las adversidades derivadas de la pobreza, violencia, desempleo, segregación y discriminación en el área laboral, no tengan las consecuencias previstas y se pueda incidir en un cambio de dirección hacia el empoderamiento.

Corroborando lo anterior, León (2001) señala que el empoderamiento de las mujeres implica un empoderamiento psicológico y emocional de los hombres, por medio del cual muchas de ellas lograrían avanzar y quitarse la coraza limitante en que las colocan los estereotipos de género. Y dado que estas relaciones se ponen de manifiesto en todos los ámbitos de la vida de las mujeres y en gran parte en relación con "el otro" (los hombres), el empoderamiento implica no sólo un cambio de los comportamientos y experiencias de ellas, sino también de su entorno en los ámbitos publico y privado. Y si asumimos que el empoderamiento de la mujer ejerce una influencia tan significativa en su entorno, un contexto adecuado para su estudio se encuentra situado en el hogar, ya que las relaciones entre sus miembros manifiestan la dinámica de género que reproduce los patrones de igualdad y de desigualdad (Bergareche, 1997, 2001).

Cuando hombres y mujeres adoptan las polaridades que componen la construcción social del género, éstas conducen a la división no nada más del trabajo sino también de las emociones (Walters et al., 1996). Ambas esferas están embarcadas en estructuras de autoridad, que alcanzando el ámbito de los símbolos y de la ideología, está gestada principalmente en las esferas privadas (familia) para consolidarse en las públicas (trabajo). Desde las ideologías que permean el ámbito privado y que consideran a las relaciones hombre-mujer como dominadas por la naturaleza encubriendo así su fundamento cultural e histórico, se acepta un supuesto destino inevitable y esencial para hombres y mujeres (Langarde y De los Ríos, 2003). De ahí la importancia de incluir a la familia en el análisis del empoderamiento de la mujer a través del trabajo, ya que constituye una esfera reproductora de la inequidad como institución 
primaria donde se establecen y mantienen relaciones cotidianas de poder basadas en una estructura jerárquica.

El debate centrado en las relaciones de poder, el conflicto y las relaciones de género en el hogar para entender la subordinación de las mujeres abrió la posibilidad de satisfacer sus necesidades partiendo de un enfoque en los hogares, comunidades e instituciones. Este debate cuestiona el modelo de desarrollo dominante y ofrece la alternativa de un desarrollo humano sostenible y equitativo (De Villota, 1999). La familia tradicional formada por un ama de casa y un proveedor está reduciendo su poder universal como referente simbólico del ámbito reproductivo. Estamos asistiendo un proceso de destradicionalización de las relaciones de pareja y/o familia (Beck, 1988; Medina, 2003) y al mismo tiempo de afirmación de las mismas, aunque sea sobre nuevas bases.

Son múltiples las raíces de esas transformaciones, entre las que figuran cambios macroestructurales donde la cara más llamativa es la reestructuración productiva a escala mundial, teniendo como antesala el proceso globalizador que afecta los referentes básicos de la vida social. Uno de estos referentes atañe a la formación de los procesos de identidad cuyas repercusiones se dejan sentir en el mundo familiar (Giddens, 2000), con la intrusión de valores y realidades culturales ajenas (globales) en la cotidianidad familiar. Además, las transformaciones económicas han ido emparejadas a crisis económicas vividas por los países más empobrecidos donde el desempleo, la precariedad laboral y la migración masiva han dado como resultado una mayor presión hacia la participación económica femenina.

Los cambios sociodemográficos anteceden también a un conjunto decisivo de cambios sociales, siendo uno de los más relevantes las transformaciones en la esfera de la reproducción sociobiológica, acortando el tiempo dedicado a ella. El control de la fecundidad en la mayoría de la población ha posibilitado una mayor autonomía de las mujeres sobre sus cuerpos, acentuando la separación entre la reproducción y la sexualidad. Este aspecto representa un cambio de gran trascendencia para la concepción de vida de pareja/familia, pues al independizar a las mujeres de la sujeción a las condiciones de reproducción natural, les abre la posibilidad de optar por otros itinerarios sociales.

Otros rasgos que han tenido impacto en el mundo de pareja/familia son una serie de transformaciones de carácter cultural que de manera directa o indirecta han culminado en la modificación de las expectativas de lo que son o deben ser las mujeres, dando pie a la construcción de nuevas imágenes de la feminidad menos centradas en la maternidad (Burin, 2001). En este cambio 
inciden un número importante de sucesos: la entrada masiva de las mujeres a la educación superior, la creciente urbanización, la exposición continua a otras culturas vía los medios de comunicación de masas, el creciente individualismo, el control de la fecundidad y la cada vez mayor incorporación de la mujer a la actividad económica, entre otros.

Todos los elementos que se han señalado han dejado huella en la configuración y dinámica al interior de las familias. Sin embargo, en esta sección centraremos nuestra atención en dos puntos particulares y su relación con los procesos del turismo: 1. La familia como unidad de análisis para entender la condición femenina; 2 . Los cambios en la dinámica y organización de la pareja/familia seguidos a la incorporación de la mujer al mercado de trabajo y su vinculación con el empoderamiento.

Considerando la familia como unidad de análisis, partimos de la premisa de que redefinir a la familia como una entidad construida socialmente, además de restarle su carácter universal para forjar el progreso de los individuos que la conforman, abrió las puertas para atender el espacio privado donde ésta se desarrolla. Anteriormente, aludiendo al carácter natural o divino de su creación, se menospreciaba la atención que se debía dar a la misma bajo el supuesto de que la familia era un sitio seguro donde se brindaba nutrición emocional a sus miembros (valoración, respeto, amor), además de seguridad para su desarrollo y crecimiento. Según Medina (2000), un buen número de estudios en torno a la familia coincidían en que la familia nuclear patriarcal era el modelo a seguir por las sociedades modernas al armonizar con los requerimientos de la economía industrial. Se señalaron como sus funciones básicas la sexualidad, la reproducción, la socialización y la economía.

A partir de esta concepción estructural y funcional de la familia, se definieron implícitamente los roles parentales y maternales. Se describió al esposo como un líder instrumental, y a la esposa como un líder expresivo (Parsons, citado en Medina, 2000), dicotomía que conlleva relaciones de poder asimétricas influidas por el género. Estos roles polarizados han servido para mantener la homeostasis o armonía de un sistema que se traduce en una división de trabajo acorde a las características que hombres y mujeres han construido. Por ejemplo, las mujeres son definidas como dependientes de la conducta masculina y esa noción tiene el efecto de mantenerlas estáticas en una etapa psicológica.

No cabe duda que la dependencia significa una amenaza mucho más grave a la posibilidad de supervivencia que la autonomía-separación definidos como 
atributos masculinos. El imaginario social de éxito y el logro es masculinizado y adoptado tanto por hombres y mujeres, teniendo como escenario los espacios públicos interpretados como la cuna del desarrollo individual y social a través de oportunidades laborales y comerciales, prestigio y dinero. Este discurso, adherido a la estructura social y cultural, se apropia de las identidades, expresándose y reproduciéndose en ámbitos tan particulares como el hogar. De ahí que aún cuando la mujer acceda al trabajo remunerado (espacio público), si en su identidad social como mujer predomina el valor de la subordinación y la dependencia, no influirá sustancialmente en el establecimiento de reglas y procesos públicos de donde emanan las relaciones de poder.

Un mérito del feminismo fue poner en tela de juicio el carácter estable de la familia como una entidad natural, lugar de cuidado, protección y desarrollo de sus integrantes (Barret y Phillips, 2003; Ariza y de Oliveira, 2001). Si bien estas cualidades son inherentes a un buen número de familias, la otra cara de la moneda es que la familia también es cuna de desigualdad e inequidad. Es el lugar donde nacen y se consolidan los prejuicios respecto al género, donde se socializan y aprenden los distintos roles (en prejuicio generalmente de las mujeres), bajo un discurso totalizador que restringe la vida de los hombres y mujeres. Estos discursos oprimen, truncan y eliminan formas alternativas de autodefinirse y relacionarse.

La redefinición de la familia que se propone desde el análisis feminista incluye un conjunto de relaciones de cooperación, intercambio, poder y conflicto que hombres y mujeres de diferentes generaciones establecen en su interior. Esta nueva mirada nos anima a analizar los espacios de poder que en función del género delimitan y caracterizan las formas por las que se legitima, negocia o impone la autoridad familiar. Una de las formas de poder que habían sido invisibles y que surge a la luz de esta nueva mirada teórica es la visibilidad de la violencia doméstica, como la expresión más contundente de las asimetrías que encierra el mundo familiar (Corsi, 2003; Navarro, 2000).

Lo anterior sirve de antesala para referirnos al punto dos de esta sección: los cambios en la dinámica y organización de la pareja/familia como consecuencia de la incorporación de la mujer al mercado de trabajo y su vinculación con el empoderamiento. Según Arriagada (1994: 158):

[...] han ido surgiendo algunas dimensiones de la modernidad como una nueva definición de los roles conyugales, en la cual el principio de igualdad se manifiesta lentamente y se relaciona con el aporte económico de mujeres e hijos al hogar. 
Hay nuevas relaciones entre padres e hijos que reflejan un mayor reconocimiento de los derechos de los niños y un debilitamiento de las relaciones de jerarquía y sumisión.

Sin embargo, también se documenta que los roles asociados con el desempeño de los trabajos reproductivos (labores domésticas y crianza de los hijos) han sido menos susceptibles al cambio (Badinter, 2003; Bonino, 2002). A pesar de las transformaciones registradas en las generaciones más jóvenes y en los sectores sociales más educados, la esposa sigue siendo, en la mayoría de los casos, la responsable de la supervisión y/o realización de las tareas domésticas. La participación de los varones asume con frecuencia la forma de ayuda esporádica los fines de semana, en las vacaciones y en casos de enfermedad $y$, con mayor regularidad, cuando las cónyuges desempeñan actividades extradomésticas remuneradas (Ariza y de Oliveira, 2001).

Se ha notado un incremento mayor de la participación de los hombres en actividades de cuidado y recreación de los niños, reparación de la casa y trámites administrativos, que en labores domésticas tales como limpiar la casa, lavar, planchar y cocinar (Rojas, 2007). En general las amas de casa de los sectores más pobres han delegado una mayor responsabilidad y carga de trabajo en sus hijos, ante la necesidad de incorporarse al trabajo asalariado, lo que no significa la desaparición de la doble jornada (González; 2000). Esta necesidad también está siendo asociada a cambios conductuales y la sobrecarga que implica se relaciona con problemas como depresión, ansiedad y enfermedades psicosomáticas entre otras (De la Fuente, 1997).

Aparece una contradicción gestada al interior de los hogares, manifestándose en que por un lado se ha forzado a las mujeres a participar en el trabajo remunerado sin haberse liberado de sus responsabilidades domésticas y por el otro no han cambiado las normas y valores asignados culturalmente a mujeres y hombres. El conflicto entre el deber ser y el hacer de hombres y mujeres se ha recrudecido y se pretende que se llegue a soluciones de solidaridad y consenso más que a situaciones de confrontación y violencia. Los estudios sobre trabajo y empoderamiento de la mujer en el contexto del desarrollo han alcanzado particular relevancia para entender estos procesos durante las últimas dos décadas, poniendo en evidencia la estrecha relación de éstos con aspectos tales como bienestar individual y familiar, salud y desarrollo social (Casique, 2007; Pick, 2007; De Oliveira y Ariza, 1999; García y De Oliveira, 1998; León, 2001; Bergareche, 2001, 2004, 2006). 
Sin embargo, la relación entre trabajo femenino remunerado y el empoderamiento arroja resultados contradictorios. Algunos estudios, tanto en México como en otros países, sugieren que en general las mujeres que trabajan remuneradamente reportan un mayor nivel de poder en la toma de decisiones familiares (Ariza y De Oliveira 2007; González, 2000). En los sectores populares, los resultados no son tan optimistas. Cuando las cónyuges reciben ingresos similares o superiores, el varón puede sentir amenazado su papel de proveedor principal, su autoridad en la familia y su masculinidad; situación que con frecuencia desencadena una mayor opresión y violencia hacia las mujeres. Esto ocurre más en las familias donde las mujeres casadas son las jefas económicas (Casique, 2007; Zapata et al., 2002).

Por otra parte, algunos estudios reportan que la violencia doméstica tiende a disminuir cuando las mujeres pueden contribuir económicamente al hogar, alcanzan cierto nivel educativo y tienen participación en redes sociales (Ariza y De Oliveira 2007; García, 2007). Otros estudios, en cambio, sugieren un exacerbamiento de la violencia doméstica cuando las mujeres generan su propio ingreso (Ramírez, 2005). Los resultados de estas investigaciones ponen de manifiesto la importancia de seguir abordando la relación entre violencia, trabajo y empoderamiento para identificar patrones significativos que marquen pautas hacia algunas respuestas.

Cito la investigación realizada en México que examina los resultados del Programa Oportunidades, operacionalizado tras la idea de que el manejo de los recursos económicos por las mujeres llevaría a un mayor bienestar familiar, aumentando los niveles de poder de las mismas. La interpretación optimista sugiere que se cumplió el objetivo para el que fue implementado el programa, ya que su implementación refleja una mayor autonomía y poder de decisión. Así mismo, la educación recibida se tradujo en más seguridad y aumento de autoestima en las mujeres (Scout, 1999; Sedesol, 2003; Briere, Mindek y Quisumbing, 2000; Sedesol, 2003). Sin embargo, tres de las cuatro investigaciones citadas concuerdan en señalar que ha habido "efectos secundarios", en especial, la intensificación de la tensión intrafamiliar.

Por ejemplo, Briere, Mindek y Quisumbing (2000: 79) refieren que “...se generan consecuencias no previstas, como un incremento en las tensiones sociales dentro del hogar". Otros datos relevantes refieren reportes de violencia, más no pueden especificar que el programa sea el origen de ésta: "es más probable que los requisitos del Programa puedan provocar casos de violencia en hogares en los que ya existía". Sin embargo, Sedesol (2003) reporta que en 
las relaciones domésticas no hubo una tendencia específica, señalando separaciones conyugales, abandonos familiares por parte de algunos hombres y disminución de aportaciones que éstos hacían hace un año al presupuesto hogareño. Scout (1999), por medio de su encuesta de opinión a las beneficiarias del programa, encuentra que solamente $5 \%$ se quejaron de un efecto negativo por problemas con su esposo y menor ayuda por parte de sus hijos.

Una posible limitante teórica que podría explicar la variabilidad en los resultados, además de probables sesgos de orden metodológico, es que las investigaciones citadas parten de la idea de que el dinero es un instrumento de poder en las sociedades presentes. Sin embargo, por sí sólo el dinero no representa poder para las mujeres trabajadoras, ya que tiene que estar ligado a ideologías de género que le otorguen dicho significado. Por ejemplo, ganar dinero para las mujeres significa muchas de las veces mejoras para la familia, y en situaciones de pobreza contribuye a la sobrevivencia. Sin embargo, la individualidad de la mujer se disuelve en su entrega a las necesidades del grupo doméstico, generalmente subordinando a él su consumo particular.

Bergareche (2006) presta atención a los factores culturales y simbólicos en la relación trabajo, violencia y empoderamiento en Ciudad Juárez, mostrando cómo la aportación económica de la mujer al hogar u otros factores de tipo material no son tan relevantes para el empoderamiento —entendido como ausencia de violencia sexual - como la lente simbólica del rol laboral a través de aspectos como la espiritualidad (Bergareche, 2006). Los hombres, en cambio, gestionan el dinero desde la seguridad que proporciona el saberse proveedores libres. Su identidad personal sigue construyéndose desde el modelo interiorizado de la masculinidad tradicional.

Por lo tanto, haciendo eco del argumento del GED, el poder se sitúa más allá del control de los recursos económicos y está articulado a las ideas y creencias que determinan las reglas y los ideales, influido sobre todo por factores culturales y sociales. Las ideas que hombres y mujeres han construido sobre su "deber ser" son el mapa que se ajustará en su identidad. De ahí la importancia de colocar la interpretación y mediación de las experiencias laborales de las mujeres en el centro de las indagaciones el poder a través del trabajo (Bergareche, 2006). Este punto de partida puede utilizarse como escenario tanto para retar los roles tradicionales, como para dar poder a las mujeres trabajadoras en términos económicos, sociales, psicológicos y políticos. Si la industria del turismo transformara las relaciones tradicionales de género en el trabajo conllevaría a inevitables rupturas de los modelos de identidad de 
ambos sexos, poniéndose de manifiesto en todas las áreas de su vida cotidiana. Esto tendría implicaciones en cuestiones de dominación, y por lo tanto de poder, que potencialmente conducirían hacia una mayor igualdad de género al interior de los espacios privados.

\section{Conclusión}

El presente ensayo ha tratado en primer lugar de revisar los conceptos que guían el debate sobre género y desarrollo como base teórica de la situación de la mujer en el contexto del desarrollo turístico. Se pudieron identificar las bondades y limitaciones de las principales vertientes teóricas, mostrando la necesidad de reorientar el debate hacia aspectos más relevantes a la realidad local, cotidiana y simbólica de la participación de la mujer en el turismo. Dicha propuesta surge de la necesidad de ampliar las vertientes de investigación y de identificar las áreas de poder y transformación de las mujeres protagonistas del turismo, áreas que se debilitan o anulan al ser posicionadas frente a los aspectos explotativos y marginales de las estructuras protagonistas del desarrollo a nivel macro social.

Partiendo de esta premisa, en segundo lugar se identificaron áreas del empoderamiento que no han sido suficientemente exploradas en el paradigma sobre trabajo, poder y desarrollo, haciendo especial énfasis en el ámbito de las emociones asociadas con sus roles de género y con un impacto directo en procesos de empoderamiento y victimización de las mujeres. Dado que esta interacción entre construcción social, emoción y poder/victimización se manifiesta más activa e intensamente en el ámbito familiar por ser la cuna social de la expresión de las emociones, se reivindica y articula el contexto familiar como laboratorio social especialmente adecuado para explorar esta relación y los elementos inherentes a ella. Así mismo, como complemento a lo anterior, se propone la exploración de los aspectos más subjetivos de la experiencia laboral a través de la identificación de significados asociados a la identidad local y las narrativas de lo cotidiano, incluyendo el rol de las emociones y su relación con el hogar.

La incorporación de los elementos mencionados al debate sobre género, turismo y desarrollo en el Pacífico mexicano puede no sólo enriquecer el debate teórico sobre el tema, sino también optimizar de forma significativa los elementos de empoderamiento de las mujeres protagonistas del quehacer cotidiano en el contexto del turismo. De esta manera, la investigación en 
esta área cumplirá un objetivo vital en la transformación y el bienestar de las protagonistas de estos procesos y su entorno cotidiano, apuntado a vías productivas hacia el cambio social, la equidad y la calidad de vida de mujeres, hombres y familias en la Cuenca del Pacífico mexicano. આญ

\section{Bibliografía}

Aitchison, C. (2001), "Theorizing other discourses of tourism, gender and culture: can the subaltern speak (In tourism)", Tourist Studies, 1 (2): 133-147.

Ariza, M. (2000), "Ya no soy la que dejé atrás... Mujeres migrantes en República Dominicana”, Instituto de Investigaciones Sociales, México: UNAM/ Plaza y Valdés.

Ariza, M. y De Oliveira (2007), "Familias, pobreza y desigualdad social en Latinoamérica: una mirada comparativa”, en Estudios Demográficos y Urbanos, 22, (1), enero-abril.

Ariza, M. y De Oliveira, O. (2001), "Familias en transición y marcos conceptuales en redefinición”, Papeles de Población 28, 9-39.

Arriagada, I. (1994), "Transformaciones del trabajo femenino urbano", CEPAL $53,18-35$.

Badinter, E. (2003), "Hombres / Mujeres. Como salir del camino equivocado", Argentina: Fondo de Cultura Económica.

Banco Mundial (2006), "Hacia la integración de género en el desarrollo económico mediante la igualdad de derechos", Banco Mundial: Alfaomega.

Barret, M. y Phillips, A. (2003), Desestabilizar la teoría: Debates feministas contemporáneos, México: Paidós.

Bauman, Z. (1999), La globalización: consecuencias humanas, México: Fondo de Cultura Económica.

Sen, A. (2002). "La misoginia como problema de salud pública", Letras libres, 4. 40, 12-18.

Beck, U. (1988), La sociedad en riesgo: Hacia una nueva modernidad, Barcelona: Paidós.

Beneria, L. (2005), Género, desarrollo y globalización, Barcelona: Hacer Editorial.

Bergareche, A. (1997), "Reshaping empowerment: the relevance of violence in the new international division of labor debate", Frontera Norte 17, vol. 9, enero-junio, Colef, Tijuana. 
Bergareche, A. (2001), “Los límites de la autonomía: la asimilación del discurso católico en la conceptualización de la violencia intrafamiliar”, Los rostros de la violencia, Colef, Tijuana.

Bergareche, A. (2001), "Interpreting autonomy: work, sexual violence and women's empowerment in the Northern Mexican border", tesis doctoral, Universidad de Londres, Londres.

Bergareche, A. (2004), "Tiempos de ambivalencia: discurso político, violencia e identidad laboral en Ciudad Juárez”, en Argelia de la Torre Barrón, Rosina Ofeda Cárdenas y Carlos Javier Maya Ambía (coords.), Construcción de género en sociedades con violencia. Un enfoque multidisciplinario, México: Porrúa.

Bergareche, A. (2006) "The roots of autonomy through work participation in the Northern Mexico border region", en Doreen Mattingly y Ellen Hansen, Women and Change at the Us-Mexico Border: Mobility, Labor and Activism, Tucson: University of Arizona Press.

Bishop, R. y Robinson, L. (1998), Languages of tourism. Night market, economic miracle, Nueva York: Routledge.

Boletín Hechos y Tendencias del Turismo (2007), Tendencias Mundiales del Empleo de las Mujeres, en: www.sectur.gob.mx/wb2/sectur/sect_Estadisticas_del_Sector.

Bonino, M. (2002) "Los varones ante el problema de la igualdad con las mujeres”, en Lamas, C. (ed.), ¿Todos los hombres son iguales? Identidad masculina y cambios sociales, Barcelona: Paidós.

Boserup, E. (1970), Women's Role in Economic Development, Nueva York: St. Martins.

Byrne S. (2005) "Las dimensiones de género en la investigación sobre turismo: temas globales, perspectivas locales", Política y sociedad, 42 (1): 25-37.

Brière, B., Mindek, D. Quisumbing, A. (2000). Progresa Programa de educación, salud y alimentación. México, en: http://www.fao.org/Regional/ LAmerica/prior/desrural/pobreza/progresa.pdfsed.

Casique, I. (2007), "El complejo vínculo entre empoderamiento de la mujer y violencia de género", en Castro R y Casique I. (edit.), Estudios sobre cultura género y violencia contra las mujeres. México: UNAM.

So-Min Cheong y Marc L. Miller (2000), "Power and tourist: a foucauldian observation", Annals of Tourism Research 72 (2): 371-390

Cohen, E. (2002), "Authenticity, equity and sustainability in tourism”, Journal of sustainable Tourism 10(4): 267-276. 
Corsi, J. (2003), Maltrato y abuso en el ámbito doméstico, Argentina: Paidós.

De la Fuente J. (1997), Salud mental en México, México: Fondo de Cultura Económica.

De la O Martínez, Ma. (2000), "Las mujeres en la reestructuración: Implicaciones para Canadá, Estados Unidos y México”, en María Luisa González M. (coord.), Globalización en México y desafíos del empleo femenino, México: UNAM/Porrúa.

De la O Martínez, Ma. (2001), "Flexibilidad, trabajo y mujeres. Ausencia y presencia en los estudios de trabajo en México, 1988-1998”, Revista Región y Sociedad, El colegio de Sonora, vol. XII, núm. 19, pp. 85-100.

De Oliveira, O y Ariza, M. (1999), “Trabajo, familia, y condición femenina. Una perspectiva de análisis”, Papeles de Población 20, 89-129.

De Villota, P. (1999), Globalización y género, Madrid: Editorial Síntesis.

Deere, C. (1977), "Changing relations of production and Peruvian peasant women's work", Latin American Perspective 4, 88-96.

Droeven, M., Andolfi M., Swarstein J. (1997), Más allá de pactos y traiciones, Argentina: Paidós.

Enloe, C. (1999), Bananas, beaches and bases: Making feminist sense of international politics, Berkeley: University of California Press.

Enloe, C. (2000), Maneuvers: the international politics of militarizing women's lives, Berkeley, Londres: University of California Press.

Falquet, J. (2003), "Mujeres, feminismo y desarrollo. Un análisis crítico", Desacatos. Revista de Antropología Social. México: 11:13-35.

García, B. y De Oliveira, O. (1998), "Trabajo femenino y vida familiar en México”, México: El Colegio de México.

García, B. (2007), “Cambios en el trabajo femenino en México", Papeles de población, México: UAEM. Julio-septiembre, núm. 53, pp. 23-45.

García, V. (2004), "Mujer, derechos, realidad y desarrollo social”, en Alberto Hidalgo y Raúl Medina (coords.), Cooperación al desarrollo y bienestar social, Oviedo: Universidad de Guadalajara, Instituto de Estudios para la Paz y la Cooperación.

Gatens, M (2004), "El poder, los cuerpos y la diferencia", en Barret M. y Phillips, A. (coords.), Desestabilizar la teoría. Debates feministas contemporáneos, México: Paidós.

Giddens, A. (2000), Consecuencias de la modernidad, Madrid: Alianza Editorial. 
González, M. (2000), Globalización en México y desafíos del empleo femenino, México: UNAM-IIE, DGAPA.

Hidalgo, C. (1998), "El pensamiento económico sobre el desarrollo, de los mercantilistas al PNUD", España: Universidad de Huelva.

Índice de equidad de género avances y retrocesos, en: http://www.socialwatch. org/es/avancesyRetrocesos/IEG_2008/tablas/Brechaeneducacion.htm.

Kabeer, N. (2004), Realidades trastocadas. Las jerarquías de género en el pensamiento del desarrollo, México: Paidós.

Kinnaird, V. y Hall, D. (1994) "Understanding tourism processes: A genderaware framework", Tourism Management 17(2): 95-102.

Langarde, M. y De los Ríos, M. (2003), Los cautiverios de las mujeres: madresposas, monjas, putas, presas y locas, México: UNAM.

León, M. (2001), "Empoderamiento de las mujeres: encuentros del primer y tercer mundo en los estudios de género", La Ventana, Universidad de Guadalajara, 13: 94-116.

López, I. y Alcalde, R. (1999), Relaciones de género y desarrollo. Hacia la equidad con cooperación, Madrid: Instituto Universitario de Desarrollo y Cooperación.

Marchand, M. (2002), Engendering globalization in an era of transnational capital. New cross-border alliances and strategies of resistance in a post-NAFTA Mexico, Kriemild Saunders (ed.), Londres: Zed Books.

Marrero, S. (2005) "Repercusiones del neoliberalismo y la globalización en el trabajo y la mujer trabajadora", Revista Cubana de Salud y Trabajo 6(2): 65-68.

Medina, A. (1993), "Puerto Vallarta de Pueblo a Ciudad", en Olvera Jaime (ed.), Una aproximación a Puerto Vallarta, Zapopan, Jalisco: El Colegio de Jalisco.

Medina, L. (2003), "Commoditizing Culture: Tourism and Maya Identity", Annals of Tourism Research 30 (2): 353-368.

Medina, R. (2000), "Contextos y circunstancias de la nueva paternidad", Perspectivas Sistémicas: 13, (63) 3-7.

Melillo, A., Soriano, R., Méndez, J. y Pinto, P. (2004), "Salud comunitaria, salud mental y resiliencia”, en Melillo, Suárez y Rodríguez (comps.), Resiliencia y subjetividad, Argentina: Paidós.

Moira, G. (2004) "El poder, los cuerpos y la diferencia”, en Barret, M. y Phillips, S (coords.), Desestabilizar la teoría. Debates feministas contemporáneos, México: Paidós.

Monreal, P. (1999), "Mujeres, género y desarrollo, conceptos y mundos encontrados”, en Gimeno, J. y Pilar, M. (coords.), La controversia del desarrollo. 
Crítica desde la antropología, Madrid: Instituto Universitario de Desarrollo y Cooperación.

Moreno, L. y Lunar, L. (2006), “Turismo y género: empleo de la mujer en la actividad turística en la Isla Margarita, estado Nueva Esparta”, PASOS Revista de Turismo y Patrimonio Cultural 4 (3): 373-389.

Moser, C. (1995), Planificación de genero y desarrollo. Teoría, práctica y capacitación, Lima: Red entre Mujeres y Ediciones Flora Tristán.

Navarro, G. (2000), “La pareja violenta”, en Navarro, G. y Pereira, M. (comps.), Parejas en situaciones especiales, Barcelona: Paidós.

Organización Internacional del Trabajo (2001), "En busca de un turismo socialmente responsable”, en: http://www2.ilo.org/public/spanish/bureau/ inf/magazine/39/tourism.htm.

Oficina Internacional del Trabajo (2008), "Tendencias mundiales del empleo de las mujeres”, en: http://www.oit.org.mx/pdf/publicaciones/tendencias_mundiales_mujeres.pdf.

Payne, M. (2002), Terapia narrativa, Barcelona: Paidós.

Pérez, de C. (2006) "Nuestra diversidad creativa. Informe de la comisión mundial de cultura y desarrollo", Montevideo: UNESCO.

Pick, S. Sirkin, J, Ortega, I. et al. (2007), "Escala para medir agencia personal y empoderamiento (ESAGE)", Rev. interm.psicol. 41(3): 295-304.

Plan Municipal de Desarrollo 2004-2006. H. Ayuntamiento de Puerto Vallarta.

Ramírez, J. (2005), Madejas entreveradas. Violencia masculinidad y poder, México: Editorial Plaza y Valdés.

Rendón, T. (2003), “Género, desarrollo y cooperación al desarrollo”. Documentos de Trabajo en Análisis Económico. México: UnAM, 4 (2) 1-16.

Rojas, O. (2007), "Paternidad y vida familiar en la Ciudad de México", México: El Colegio de México.

Romero, R. (2006), "La división sexual del trabajo en el pensamiento feminista: evolución y retos”, en Amelia Valcárcel, Ma Dolores Renau y Rosalía Romero (comps.), Los desafíos del feminismo ante el siglo XXI, España: Instituto Andaluz de la Mujer.

Salazar, N. (2006), "Antropología del turismo en países en desarrollo: análisis crítico de las culturas, poderes e identidades generadas por el turismo", Tábula Rasa, Bogota, Colombia, 5 (2) 99-128.

Scout, J. (1999), “Análisis del programa de educación, salud y alimentación”, programa Oportunidades, México. 
Sedesol (2003), "Evaluación cualitativa del programa de desarrollo humano Oportunidades".

Sen, A. (2002), "La misoginia como problema de salud pública", Letras libres, 4. $40,12-18$

Sluzky, C. (2003), La red social. Frontera de la terapia sistémica, Barcelona: Paidós.

Sluzky, C. (2006), "Violencia familiar y violencia política. Implicaciones terapéuticas de un modelo general”, en Dora Fried (coord.), Nuevos paradigmas, cultura y subjetividad, Argentina: Paidós.

The Global Gender Gap Report (2007), World Economic Forum, en: http:// www.weforum.org/pdf/gendergap/report2007.pdf.

Townsend, J. (2002) "Cómo entender el poder", en Zapata, E., Townsend, J., Rowlands, J., Manzanares, P., González M. (comps.), Las mujeres y el poder, México: Plaza y Valdés.

Vargas, E. (2009), "Desarrollo, género y empoderamiento en el contexto del turismo", Tesis doctoral (en proceso), UdeG, Cedestur, Centro Universitario de la Costa, Puerto Vallarta.

Velázquez, G. (1993), "Puerto Vallarta, ciudad de atracción migratoria", en Olveda, J. (ed.), Una aproximación a Puerto Vallarta, Zapopan, Jalisco: El Colegio de Jalisco.

Venegas, P. (2006), "El empoderamiento de las mujeres. Un camino hacia la equidad y el desarrollo", en De Sur a Sur, Revista Andaluza de Solidaridad, Paz y Cooperación 31, enero- febrero.

Villaseñor, P. (1993), “Turismo y desarrollo regional”, en Olveda, J. (ed.), Una aproximación a Puerto Vallarta, Zapopan, Jalisco: El Colegio de Jalisco.

Walters M., Carter, B., Papp, P., Silvesrtein, O. (1996), La red invisible. Pautas vinculadas al género en las relaciones familiares, Barcelona: Paidós.

Watzlawick, P. (1989), La realidad inventada, Barcelona: Gedisa.

White, M. (1994), Guías para una terapia familiar de sistema, Barcelona: Gedisa.

White, M. y Epston, D. (1993), Medios narrativos para fines terapéuticos, Barcelona: Paidós.

Zapata, E., Townsend, J., Rowlands, J, Manzanares, P., González M. (2002), Las mujeres y el poder, México: Plaza y Valdés. 